\title{
Optical coherence computed tomography for quantitative 3D imaging of adult zebrafish (Withdrawal Notice)
}

Jelle Van der Horst, Anna Trull, Jeroen Kalkman

Jelle Van der Horst, Anna Trull, Jeroen Kalkman, "Optical coherence computed tomography for quantitative 3D imaging of adult zebrafish (Withdrawal Notice)," Proc. SPIE 10499, Three-Dimensional and Multidimensional Microscopy: Image Acquisition and Processing XXV, 104991D (21 March 2018); doi: 10.1117/12.2287856

SPIE. Event: SPIE BiOS, 2018, San Francisco, California, United States 


\section{Optical coherence computed tomography for quantitative 3D imaging of adult zebrafish (withdrawal notice)}

Jelle Van der Horst, Anna Trull and Jeroen Kalkman

Technische Univ. Delft (Netherlands)

Proc. SPIE 10499, 104991D(2018)

Online Publication Date: 14 March 2018

Withdrawn from Publication: 21 March 2018

Conference Date: 29-31 January 2018

Conference Location: San Francisco, California, United States

Conference Title: Three-Dimensional and Multidimensional Microscopy: Image Acquisition and

Processing XXV

Conference Chairs: Thomas Brown, Carol Cogswell, Tony Wilson

Publisher's Note: This paper, originally published on 14 March 2018, was withdrawn per author request. 